\title{
IMPACTO DE LOS CRÉDITOS EN LAS GANANCIAS DE LAS MICROEMPRESAS EN COSTA RICA ${ }^{1}$
}

\author{
IMPACT OF CREDIT ON MICRO-ENTERPRISE PROFIT IN COSTA RICA
}

IMPACTO DOS CRÉDITOS SOBRE OS LUCROS DAS MICROPRESAS NA COSTA RICA

\section{Carmen Sánchez Retana²}

\begin{abstract}
Resumen
Esta investigación tiene como objetivo evaluar el impacto de los créditos en las ganancias de las microempresas de Costa Rica. Para ello se utilizan los datos de la ENHOPRO 2015 y se desarrolla la metodología Propensity Score Matching. Los resultados obtenidos demuestran que los créditos tienen un impacto positivo y estadísticamente significativo en las ganancias de las microempresas de los hogares en Costa Rica. Es decir, la obtención de financiamiento por parte de las microempresas contribuye en el crecimiento de las ganancias de estas.
\end{abstract}

Palabras claves: microcréditos; empresas; emprendimientos; Propensity Score Matching; microfinanzas

\begin{abstract}
The objective of this research paper is to evaluate the impact of credit on the profit of microenterprises in Costa Rica. ENHOPRO 2015 data is used together with the Propensity Score Matching methodology. Results show that credit has a positive and statistically significant impact on the profit
\end{abstract}

DOi: https://doi.org/10.15359/eys.24-55.1

Fecha de recepción: 21-11-2018. Fechas de reenvíos: 18-12-2018, 26-12-2018. Aceptado el 26-12-2018. Publicado el 01-01-2019.

${ }^{1}$ Este trabajo es una adaptación del proyecto de graduación para optar por el grado de Máster en Finanzas, Banca y Seguros, de la Universidad de Málaga, España.

2 Máster en Finanzas, Banca y Seguros de la Universidad de Málaga (España) y Licenciada en Economía de la Universidad de Costa Rica (Costa Rica). Ha ejercido como investigadora en el Instituto de Investigaciones en Ciencias Económicas (IICE-UCR) de la Universidad de Costa Rica (UCR) y analista en entidades públicas y privadas. Correo electrónico: csanchezretana@uma.es ORCID: https://orcid.org/0000-0001-5185-7328

Carmen Sánchez Retana

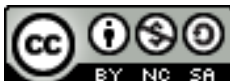

Revista Economía y Sociedad by Universidad Nacional is licensed under a CreativeCommons Reconocimiento-NoComercial- 
of microenterprises in Costa Rican homes. Consequently, financing obtained by microenterprises contributes to increase their profit.

Keywords: microcredits; business; ventures; Propensity Score Matching; microfinance

\section{Resumo}

O objetivo desta pesquisa é avaliar o impacto dos créditos sobre os lucros das microempresas na Costa Rica. Para isso, são utilizados os dados do ENHOPRO 2015 e desenvolvida a metodologia Propensity Score Matching. Os resultados obtidos mostram que os créditos têm um impacto positivo e estatisticamente significativo nos lucros das microempresas das famílias na Costa Rica. Em outras palavras, ao obter financiamento, as microempresas contribuem para o crescimento de seus lucros.

Palavras-chave: microcréditos; empresas; empreendimentos; Propensity Score Matching; microfinanças

\section{Introducción}

Las microfinanzas se han caracterizado por su participación positiva en el crecimiento de las sociedades. Dentro de sus productos o servicios financieros destacan los microcréditos, los cuales son préstamos orientados a personas o sectores que, por sus características o estado de insolvencia, normalmente son excluidos del sistema financiero tradicional.

Dentro de los grupos beneficiarios de los microcréditos se encuentran los emprendimientos y las microempresas, las cuales han demostrado ser relevantes para el desarrollo económico y social de una nación -por su capacidad de generación de ingreso, empleo y tejido empresarial-

Sin embargo, es escasa la evidencia empírica que mide el verdadero efecto de los créditos otorgados a las microempresas. Las evaluaciones rigurosas iniciaron hace pocos años y su aplicación en el campo de los microcréditos es totalmente nueva (Larru, 2008). En el caso de Costa Rica, la inexistencia de investigaciones en el tema ha impedido verificar si los créditos obtenidos por los entes emprendedores se han traducido en alguna medida de bienestar para estos mismos.

Este escenario, aunado a una situación microempresarial costarricense necesitada de mayor inclusión financiera, motiva a que el objetivo de la presente investigación sea evaluar el impacto de los créditos en las ganancias de las microempresas de hogares en Costa Rica. Dicha estimación permite conocer si los créditos son fundamentales para los grupos emprendedores y sus

2

Carmen Sánchez Retana

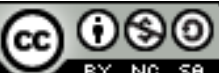

Revista Economía y Sociedad by Universidad Nacional is licensed under a CreativeCommons Reconocimiento-NoComercial- 
microempresas o si, por el contrario, se deben canalizar esfuerzos en otro tipo de productos o servicios financieros.

Para el desarrollo de la evaluación se acude a los datos de la Encuesta Nacional de Hogares Productores de Costa Rica (ENHOPRO) del año 2015 (Instituto Nacional de Estadística y Censos de Costa Rica [INEC], 2015c), y se desarrolla la metodología emparejamiento por puntajes de propensión (Propensity Score Matching en inglés).

\section{Microempresas de hogares en Costa Rica}

El informe de resultados generales de la ENHOPRO ${ }^{3}$ 2015, realizado por el INEC, señala que en dicho año se desarrollaron 371191 microempresas o emprendimientos de hogares ${ }^{4}: 69,2 \%$ de la zona urbana y el 30,8\% restante de la zona rural (INEC, 2015a).

Dichas microempresas se desarrollan en los hogares productores y son dirigidas por personas que trabajan de forma independiente. Además, se relacionan normalmente con el sector informal de la economía: su fin de producción es generar empleo e ingresos, son de pequeña escala, tienen organizaciones rudimentarias y no utilizan acuerdos contractuales formales. En concreto, el INEC asocia a las empresas con el sector informal, si estas se caracterizan por no estar inscritas en el registro de propiedad como empresa, no poseer registros contables formales y no tener asignado un salario fijo (INEC, 2015a).

Respecto a las situaciones que motivaron el emprendimiento, el $46 \%$ de las unidades productivas tuvo como origen la vivencia de una necesidad por parte de las personas dueñas, entre ellas: no encontrar trabajo asalariado o no tener trabajo, deseo de disponer de mayor flexibilidad o de complementar el ingreso familiar (INEC, 2015b). Estas necesidades pueden estar vinculadas con que, para este caso, la mayoría de grupos emprendedores presentan bajos niveles de educación (INEC, 2015b), aspecto que podría influir negativamente en su capacidad de inserción al mercado laboral (Sánchez, 2015b).

Una vez que la microempresa está en desarrollo, generalmente, el acceso al financiamiento es uno de los principales problemas que enfrentan los dueños o dueñas. Esto impide el avance de proyectos que podrían resultar exitosos y, por ende, que ayudarían a impulsar la economía de la sociedad (García, 2004). Costa Rica no ha sido la excepción a dicho comportamiento, en 2015 la

\footnotetext{
${ }^{3}$ En los siguientes años recibirá el nombre Encuesta Nacional de Microempresas de los Hogares.

4 Nombrados hasta el año 2015 por el Instituto Nacional de Estadística y Censos de Costa Rica (INEC) como emprendimientos de los hogares, ya que la institución propone emigrar al término microempresas de los hogares ante el objetivo de uniformar conceptos de la institución con los del Ministerio de Economía, Industria y Comercio (MEIC).
} 
principal necesidad de los grupos emprendedores fue el acceso al crédito, seguida por el acceso a capacitaciones (señaladas por el $48 \%$ y $32 \%$ de personas emprendedoras, respectivamente).

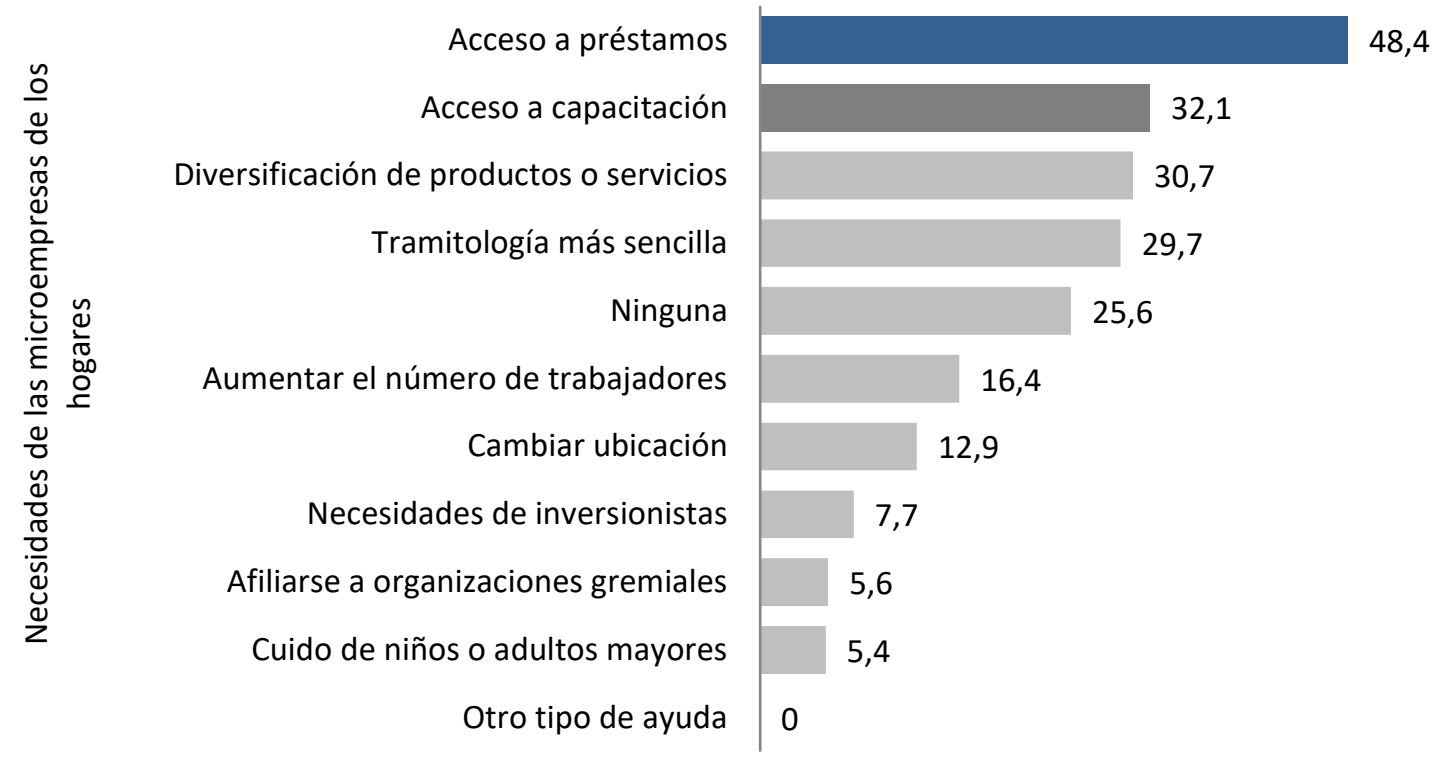

Porcentaje de microempresas

Figura 1. Porcentaje de microempresas de los hogares según necesidad requerida 5 . Año 2015. Fuente: elaboración propia con datos de INEC (2015b)

A pesar de lo anterior, los resultados de la ENHOPRO 2015 muestran que únicamente el 32\% de los hogares productores pidió algún tipo de ayuda, y apenas el 22,5\% solicitó algún crédito -porcentaje que equivale a 83367 microempresas crédito- (INEC, 2015b). Esta cifra se considera baja, si se tiene presente que el financiamiento es la principal necesidad entre la población en estudio.

De la totalidad de peticiones de ayuda, 107960 fueron favorecidas; este financiamiento es el tipo de apoyo que llegó a mayor cantidad de unidades productivas (69 327), seguido por capacitación o asesorías (47 550) y otros tipos de apoyos (11 272) (INEC, 2015b).

En cuanto a la vía por la cual se solicita apoyo, las instituciones estatales son mayormente utilizadas, por ejemplo, el $40 \%$ de la totalidad de microempresas que solicitaron algún tipo de ayuda lo hizo en un banco público (INEC, 2015c). Para el caso del financiamiento, si bien hoy en día los grupos emprendedores o las microempresas pueden solicitar créditos directos a las cuatro

\footnotetext{
${ }^{5}$ Porcentaje respecto al total de emprendimientos de los hogares. Cada emprendimiento puede tener más de una necesidad.

4 
instituciones siguientes: entidades bancarias (públicas y privadas), cooperativas y asociaciones, ONG, instituciones no formales, sus solicitudes se han dirigido principalmente a las instituciones públicas, ya sea bancos o cooperativas. En 2015, de la totalidad de microempresas que solicitaron crédito, $51 \%$ lo hizo en banca pública y $15 \%$ en mutuales o cooperativas (INEC,2015b).

Por su parte, quienes optaron por no solicitar crédito señalaron diferentes razones para no hacerlo (véase la Tabla 1), ello a pesar de que el 63,1\% de estos sí lo requería.

Tabla 1

Motivos por los que los grupos emprendedores no solicitan créditos. Año 2015. (Porcentaje de la totalidad de microempresas de los hogares que no solicitaron crédito)

\begin{tabular}{lc}
\hline \multicolumn{1}{c}{ Motivos } & Porcentaje \\
\hline No lo necesita & $36,9 \%$ \\
Desconoce el procedimiento para solicitarlo & $1,7 \%$ \\
Piensa que no se lo darían & $12,9 \%$ \\
La cuantía o el plazo no le convienen & $0,7 \%$ \\
Los intereses o comisiones son muy altos & $3,6 \%$ \\
Demasiados trámites & $6,1 \%$ \\
No le gusta endeudarse o pedir prestado & $25,7 \%$ \\
No podría pagarlo & $11,4 \%$ \\
Otra razón & $1,0 \%$ \\
\hline Total & $100,0 \%$ \\
\hline
\end{tabular}

Fuente: elaboración propia con datos de INEC (2015c).

\section{Revisión bibliográfica}

No es sino hasta los años recientes cuando ha surgido un mayor número de estudios enfocados en analizar el efecto de los instrumentos microfinancieros -aunque la calidad de estos ha sido muy variable- (Goldberg, 2005 y Rico, 2009). Ante esto, merece la pena hacer mención de los siguientes estudios, los cuales, se considera, han utilizado en sus estimaciones técnicas estadísticas robustas.

Aroca y Hewings (2009) y Sanhueza (2011) evalúan el impacto de los microcréditos en los ingresos de las microempresas en Chile. Henríquez (2009) mide el efecto de las microfinanzas en el desempeño de las microempresas chilenas. Por su parte, Augsburg, De Haas, Harmgart y Meghir (2015) evalúan para Bosnia el impacto de los microcréditos en variables como empleo, inventarios y consumo. En Costa Rica, se destaca únicamente el desarrollado por Monge y Rodríguez (2012), en el cual evalúan el impacto de servicios financieros diferentes al crédito. 


\section{Datos y metodología}

Ante el objetivo de evaluar el impacto de los créditos en las ganancias de las microempresas de los hogares, es necesario conocer cómo cambian las ganancias en dichas unidades productivas al obtener un crédito. Sin embargo, para ello, es indispensable disponer de una base muestral robusta y una metodología idónea que permita alcanzar resultados veraces.

\section{Datos}

Se utilizan los datos de la Encuesta Nacional de Hogares Productores (ENHOPRO) 2015, la cual es realizada por el Instituto Nacional de Estadística y Censos de Costa Rica (INEC, 2015c).

La unidad de análisis de la encuesta son las actividades productivas de bienes o servicios desarrollados por personas que trabajan de manera independiente y que se asocian, generalmente, al sector informal de la economía. Cabe destacar que dichas actividades productivas fueron denominadas por la institución hasta el año 2015 "emprendimientos de los hogares" y en adelante "microempresas de los hogares". También, en la encuesta se denomina "hogar productor" al hogar en donde se desarrollan dichas actividades productivas y "emprendedor" a la persona dueña del negocio. El sujeto emprendedor puede trabajar solo o en asociación a otras personas, tales como familiares o ayudantes que no reciben remuneración, 0 puede contratar personal trabajador de manera permanente o temporal, normalmente una cantidad pequeña. Específicamente se puede clasificar en: de cuenta propia (solamente la persona dueña o con ayudantes) o persona empleadora.

El periodo en estudio es el año 2015, con una muestra delimitada a 1466 microempresas, cuantía equivalente a 211037 unidades productivas a nivel nacional.

Dicho tamaño muestral es resultado de las siguientes consideraciones:

- Solamente se estudian aquellos hogares productores que desarrollan una única actividad productiva dentro del negocio, con el objetivo de no generar sesgos y poder comparar microempresas que sean lo más similares entre sí.

- La muestra es restringida a hogares productores que recibieron algún crédito para fines del negocio entre los años 2010 y 2015 . Esta delimitación permite obtener una estimación actual y precisa del impacto del crédito; la utilización de una antigüedad mayor podría afectar los resultados del efecto que se pretende analizar.

- El estudio se enfoca únicamente en los créditos brindados por instituciones financieras (bancos, establecimientos financieros de crédito, cooperativas) y organizaciones no

6 
gubernamentales. No se consideran las microempresas que solicitaron crédito al sector informal, el cual en la ENHOPRO recibe la denominación de "particulares con o sin intereses").

- No se consideran dentro de la muestra las microempresas que no solicitaron crédito al señalar que no lo necesitan. Esta restricción, la cual resalta por su alta importancia en el estudio, permite comparar microempresas caracterizadas por la necesidad crediticia, en donde la diferencia entre ellas es que unas han obtenido el crédito y otras no.

\section{Caracterización de la muestra}

Como se ha mencionado más arriba, la muestra en estudio está conformada por 1466 microempresas de hogares las cuales, según tipo emprendedor, se subdividen tal y como se aprecia en la Tabla $\underline{2}$.

Tabla 2

Microempresas según tipo de emprendedor. Año 2015

\begin{tabular}{lc}
\hline \multicolumn{1}{c}{ Tipo de emprendedor } & Número \\
\hline Cuenta propia solo & 1107 \\
Cuenta propia con ayudantes & 224 \\
Empleador & 135 \\
\hline Total & 1446 \\
\hline
\end{tabular}

Fuente: elaboración propia con datos de INEC (2015c).

En cuanto a la obtención del crédito, de la totalidad de unidades productivas que conforman la muestra, el 19,6\% obtuvo un préstamo. De dichos créditos, el $79 \%$ tuvo un valor nominal inferior a los diez millones de colones.

Al analizar las ganancias mensuales de las unidades productivas según estado crediticio (haber o no recibido préstamo), se aprecia que el $75 \%$ de las microempresas que no recibieron crédito no logran alcanzar ganancias superiores a los 277000 colones mensuales; de manera contraria, en el caso de las que sí obtuvieron crédito, el 51\% superó dicha cuantía.

A continuación, se presenta un resumen de las principales características de la muestra en estudio, tanto a nivel general como por situación crediticia. 
Tabla 3

Características de las microempresas de los hogares y sus emprendedores. Año 2015 (porcentaje de la totalidad de microempresas de la muestra en estudio)

\begin{tabular}{lccc}
\hline \multicolumn{1}{c}{ Variable } & $\begin{array}{c}\text { Total de } \\
\text { microempresas }\end{array}$ & $\begin{array}{c}\text { Microempresas } \\
\text { con crédito }\end{array}$ & $\begin{array}{c}\text { Microempresas } \\
\text { sin crédito }\end{array}$ \\
\hline Hombres & 63,64 & 78,05 & 60,14 \\
Jefe de hogar & 66,98 & 72,47 & 65,65 \\
Zona urbana & 64,26 & 54,01 & 66,75 \\
Sector agropecuario & 15,76 & 30,31 & 12,21 \\
Educación secundaria completa o más & 24,25 & 32,40 & 22,26 \\
Emprendimiento por necesidad & 49,73 & 31,01 & 54,28 \\
\hline
\end{tabular}

Fuente: elaboración propia con datos de INEC (2015c).

Como se puede apreciar en la Tabla $\underline{3}$, la participación de los hombres como dueños de las unidades productivas es mayoritaria en relación con las mujeres, aspecto que se acentúa en las microempresas en que se obtuvo crédito.

En cuanto a la proporción de jefes de hogar, el $67 \%$ de los emprendedores presentan esta función en sus hogares, aspecto que puede estar relacionado al hecho de que, como se mencionó anteriormente, la mayoría son hombres.

De la totalidad de las microempresas, el 64,3\% está ubicado en la zona urbana. Sin embargo, al hacer la distinción según situación crediticia, se aprecia que entre aquellas que recibieron crédito existe una distribución más equitativa entre zonas.

Con respecto a la educación, es baja la escolaridad de quienes componen la muestra de estudio, ya que mayoritariamente los sujetos emprendedores presentaron un nivel educativo inferior a "secundaria completa". En concreto, menos del 25\% de las microempresas se caracterizan porque sus dueños han finalizado la educación secundaria.

Por último, al hacer la distinción entre las unidades productivas que recibieron crédito y las que no, en aquellas que sí se obtuvo dicha ayuda financiera fue mayor la proporción de personas emprendedoras con al menos educación secundaria completa.

\section{Marco metodológico}

La medición del impacto de los créditos en las ganancias de las microempresas se realiza por medio de la metodología emparejamiento por puntajes de propensión (en inglés Propensity Score Matching o, por brevedad, PSM), la cual ha sido diseñada para que, por medio de una 8 
aleatorización simulada, permita estimar efectos causales de tratamiento $^{6}$ con datos no experimentales (Ordaz, 2009). En esta investigación, el tratamiento es haber recibido un préstamo para fines del negocio por parte de una entidad de crédito o una institución no gubernamental.

El objetivo de la metodología seleccionada es emparejar a participantes y a no participantes de un tratamiento con base en la probabilidad condicional de participar, dado un conjunto de características observables (Rodríguez, 2012 y Sánchez, 2015a). Khandker, Koolwal y Samad (2010) señalan que la finalidad de la metodología es encontrar dentro de los dos grupos (de tratamiento y de control) individuos similares en las características realmente importantes. Una vez establecido el emparejamiento, se realiza la comparación entre los resultados de los tratados con los no tratados y, de esta manera, se estima el correspondiente impacto medio del programa (Sánchez, 2015a).

La metodología puede exponerse en dos etapas, la primera es la estimación del puntaje de probabilidad (en inglés Propensity Score o, simplemente, PS).

El PS es un valor numérico entre cero y uno que se interpreta como la probabilidad de recibir un tratamiento (en este caso un crédito) dado un conjunto de características observables.

Por medio de este valor se resuelve el problema de dimensionalidad, ya que simplifica, en un único índice, toda la información que proporcionan diversas variables explicativas. La similitud de los individuos se refleja en dicho valor (Rosembaum y Rubin, 1984), lo que facilita encontrar pares de unidades productivas semejantes.

Inicialmente se modela la regresión binomial referente a la participación en el programa y con esta se estima, para el conjunto de individuos, la probabilidad de participar del tratamiento -dadas sus características-. En la presente, se estima el PS por medio de la modelación probit.

La forma funcional de la probabilidad de recibir el tratamiento se expresa como:

$$
p(\boldsymbol{X})=\operatorname{Pr}\{D=1 \mid \boldsymbol{X}\}
$$

Donde:

-X es el vector de características observables

-D es el tratamiento o programa

\footnotetext{
${ }^{6}$ La metodología utiliza el término tratamiento ya que sus orígenes surgieron en la rama biomédica, y si bien el método se ha expandido a diferentes ciencias el término se mantuvo.
} 
Gujarati y Porter (2010) establecen que la probabilidad de la realización de un acontecimiento está dada por:

$$
P_{i}=P(S=1 \mid \boldsymbol{X})=P\left(Z_{i} \leq \beta_{1}+\beta_{2} X_{i}\right)=F\left(\beta_{1}+\beta_{2} X_{i}\right)
$$

Donde:

- $P_{i}$ es la probabilidad estimada para cada observación $i$

- $S$ es una variable dependiente de tipo dicotómico que puede tomar los valores 1 o 0 , dependiendo de si el suceso acaece o no, respectivamente. Para efectos de este estudio $S=D$

- $Z_{i}$ es una variable normal estándar tal que $Z \sim N\left(0, \delta^{2}\right)$

Al resolver la ecuación correspondiente se obtiene la probabilidad de participación en el tratamiento (PS) para cada individuo que compone la muestra.

La segunda etapa es la estimación del efecto promedio de tratamiento en los tratados (en inglés average treatment effect on the treated, o ATT) a partir del emparejamiento entre participantes y no participantes.

Se procede al casamiento de las observaciones que han recibido el tratamiento y las que no, utilizando los puntajes estimados previamente. Una vez realizado, se calcula el ATT.

Según Becker e Ichino (2002), se puede estimar el ATT a través de la siguiente expresión:

$A T T=\mathrm{E}\left\{Y_{1 i}-Y_{0 i} \mid D_{i}=1\right\}$

$A T T=\mathrm{E}\left\{Y_{1 \mathrm{i}}-Y_{0 \mathrm{i}} \mid D_{\mathrm{i}}=1, \mathrm{p}(\boldsymbol{X})\right\}$

$A T T=\mathrm{E}\left\{\mathrm{E}\left\{Y_{1 \mathrm{i}} \mid D_{\mathrm{i}}=1, \mathrm{p}\left(X_{\mathrm{i}}\right)\right\}-\mathrm{E}\left\{Y_{0 \mathrm{i}} \mid \mathrm{D}_{\mathrm{i}}=0, \mathrm{p}\left(\boldsymbol{X}_{\mathrm{i}}\right)\right\} D_{\mathrm{i}}=1\right\}$

Siendo:

- $\quad Y_{1 i}$ el resultado si el individuo es tratado;

- $\quad Y_{0 i}$ el resultado si el individuo no es tratado.

El valor del ATT indica el impacto de los créditos en las ganancias de las microempresas de los hogares. Según Heckman, Ichimura y Todd (1998), para su estimación se acude al método emparejamiento (matching en inglés), el cual es una técnica cuasi- experimental de evaluación que se basa en la idea de contrastar los resultados de los participantes en el tratamiento con los 
resultados de los no participantes (grupo de control), se atribuye dicha diferencia de resultados al programa o tratamiento en cuestión, en este caso el préstamo recibido por el hogar productor.

El emparejamiento de los individuos que han recibido el tratamiento con los que no y la posterior determinación del ATT puede realizarse por medio de diferentes algoritmos entre los que destacan los emparejamientos por vecino más cercano (Nearest Neighbor Matching), por estratificación (Stratification Matching) y por kernel (Kernel Matching) (Becker e Ichino, 2002). En esta investigación se opta por estimar el impacto a partir de los tres algoritmos.

El emparejamiento por vecino más cercano es un algoritmo que compara el resultado que obtiene cada individuo tratado con el individuo de control cuyo PS es el más cercano (Khander et al., 2010). Una vez realizado el emparejamiento, se determina la diferencia entre cada par de unidades en la variable de medición y, con esto, es estimado el impacto sobre las ganancias como el promedio de todas las diferencias.

Según Becker e Ichino (2002), el estimador es:

$$
\begin{gathered}
\mathrm{ATT}=\frac{1}{N^{T}} \sum_{i \in T}\left[Y_{i}^{T}-\sum_{j \in C(i)} w_{i j} Y_{j}^{C}\right] \\
\mathrm{ATT}=\frac{1}{N^{T}}\left[\sum_{i \in T} Y_{I}^{T}-\sum_{i \in T} \sum_{j \in C(i)} w_{i j} Y_{j}^{C}\right]
\end{gathered}
$$

Donde:

- $\quad N^{T}$ el número de unidades tratadas en la muestra;

- $\quad C(i)$ es el conjunto de unidades de control emparejadas a la unidad tratada $i$, con un valor estimado $p_{i}$ del PS. C $(\mathrm{i})=\min _{j}\left\|p_{i}-p_{j}\right\|$;

- $\quad w_{i j}=\frac{1}{N_{i}^{C}}$ si $j \in C(i)$ y $w_{\mathrm{ij}}$ de otra forma.

En cuanto al emparejamiento por estratificación, consiste en dividir el rango de la variación del $P S$ en intervalos de forma tal que dentro de cada uno de ellos las unidades de control y las de tratamiento tengan en promedio el mismo puntaje (Sánchez, 2015b). El impacto del programa es el promedio ponderado del impacto de cada uno de los intervalos, donde la ponderación utilizada es el porcentaje de individuos tratados dentro de cada intervalo (Khandker et al. 2010; Sánchez, 2015b). 
Según Becker e Ichino (2002), en cada intervalo, la diferencia entre los resultados promedio de los individuos tratados y los de control es estimada de la siguiente manera (Sánchez, 2015b):

Donde:

$$
\operatorname{ATT}_{q}^{S}=\frac{\sum_{i \in I(q)} Y_{I}^{T}}{N_{q}^{T}}-\frac{\sum_{j \in I(q)} Y_{I}^{C}}{N_{q}^{C}}
$$

- $\quad I(q)$ es el grupo de unidades en el estrato q;

- $N_{q}^{C}$ y $N_{q}^{T}$ son los números de unidades de control y tratadas en el estrato q, respectivamente;

- $Q$ es la cantidad total de estratos.

Por último, en el emparejamiento por kernel cada una de las observaciones tratadas es emparejada con un promedio ponderado de todas las unidades de control. Las ponderaciones a utilizar son inversamente proporcionales a la distancia entre los puntajes de probabilidad de las unidades tratadas y las de control (Ordaz, 2009; Sánchez, 2015a).

$$
\mathrm{ATT}^{k}=\frac{1}{N^{T}} \sum_{i \in T}\left\{Y_{i}^{T}-\frac{\sum_{j \in C} Y_{j}^{C} K\left(\frac{P_{j}-P_{i}}{V_{n}}\right)}{\sum_{K \in C} K\left(\frac{P_{j}-P_{i}}{V_{n}}\right)}\right\}
$$

Donde:

- K es una función kernel

- $V_{n}$ es un parámetro

Cabe señalar que el correcto desarrollo de la metodología depende de las tres condiciones destacadas por Rosembaum y Rubin (1983) como necesarias para el alcance de resultados consistentes y robustos: región de soporte común, propiedad de balance e independencia condicional.

\section{Resultados}

Previo a la exposición del desarrollo del modelo es necesario hacer énfasis en ciertos aspectos que clarifican la implementación de este y, por ende, facilitan la correcta interpretación de resultados:

- Se opta por utilizar las ganancias mensuales de la microempresa como variable de impacto, es decir, sobre la que se intenta medir el impacto. Dicha variable se define como la diferencia de los ingresos y los gastos, ambos totales. Esta variable ha sido seleccionada

12

Carmen Sánchez Retana

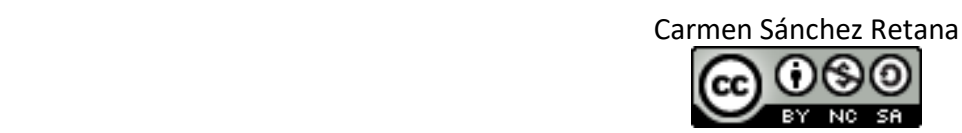

Revista Economía y Sociedad by Universidad Nacional is licensed under a CreativeCommons Reconocimiento-NoComercial- 
porque la consideración de los gastos de la unidad productiva permite dimensionar en una mejor medida el verdadero impacto del crédito en la empresa.

Asimismo, se realiza una transformación logarítmica (logaritmo natural) de dicha variable con la finalidad de brindar los resultados en términos de crecimiento porcentual

- Se considera en estado de tratamiento a aquella situación en la cual el emprendedor de la microempresa ha recibido crédito, lo cual conlleva la consideración de dos grupos:

i. el de tratados: microempresas de hogares cuyos empresarios o empresarias hayan recibido crédito;

ii. el de control: microempresas de hogares cuyos empresarios o empresarias no hayan recibido crédito ${ }^{7}$.

- Se analizan las ganancias de quienes recibieron crédito (participantes del tratamiento) en comparación a no haberlo recibido. Es una medición del impacto sobre los sujetos beneficiarios del tratamiento.

- En este tipo de estudio la decisión de participar en el tratamiento (recibir el crédito) se encuentra en manos de terceros; es decir, aunque el emprendedor o la emprendedora solicite el préstamo, no hay certeza de que lo recibirá.

\section{Estimación de los puntajes de propensión (PS)}

Para la obtención de los PS se empleó un modelo probit. Este fue controlado por diferentes variables, ya sea disponibles en la ENHOPRO o creadas a partir de estas.

\section{Variables a utilizar}

El modelo binomial asumió como variable dependiente "crédito", la cual refleja el tratamiento en cuestión. Esta misma se expresa de la siguiente manera:

$$
\text { crédito }\left\{\begin{array}{l}
1 \text { si la persona emprendedora de la microempresa recibió crédito para fines del } \\
\text { negocio } \\
0 \text { si la persona emprendedora de la microempresa no recibió crédito para fines } \\
\text { del negocio }
\end{array}\right.
$$

\footnotetext{
${ }^{7}$ Para el entendimiento de los grupos de control y de tratamiento es fundamental tener presente las consideraciones realizadas en el apartado de datos.
} 
En cuanto a las variables explicativas analizadas, a partir de los datos de la ENHOPRO se crearon aquellas que se consideraron podrían determinar la probabilidad de recibir el crédito. Con el objetivo de tener una especificación completa para cada una de las microempresas, y así poder generar posteriormente un emparejamiento adecuado, dichas variables se clasifican en tres categorías:

- Características de la actividad: zona, sector.

- Características de la microempresa: tipo de microempresa; negocio inscrito salario asignado; tenencia y número de individuos socios; empleo; ubicación del establecimiento; tipo de público comprador; tipo de venta; forma de cobro; entrega de facturas; cobro por transferencias electrónicas; tenencia de cuenta bancaria para uso exclusivo del negocio; acceso a internet; uso de teléfono fijo o celular.

- Características del sujeto emprendedor: edad, sexo, nivel educativo, tiempo desarrollo de la actividad, jefe o jefa de hogar, importancia de la actividad en los ingresos del hogar, motivo de emprendimiento, horas efectivas trabajadas la última semana.

Cabe señalar que se tuvo la precaución de considerar únicamente variables explicativas que, en su mayoría, se asume no resultan altamente afectadas en caso de recibirse el crédito.

\section{Modelación probit}

Fueron realizadas diversas estimaciones del modelo probit, para las cuales se utilizaron las distintas variables explicativas presentadas con anterioridad, esto con el fin de obtener la especificación caracterizada por el mejor ajuste y con ello alcanzar un cálculo veraz y robusto de los PS. Como criterios y medidas de ajuste para la selección del modelo se utilizaron:

- la significancia global del modelo;

- el pseudo- $\mathrm{R}^{2}$ de Mc Fadden's;

- el criterio de información de Akaike $(A / C)$;

- el criterio de información bayesiano $(B / C)$;

- el número de iteraciones para el alcance del mayor valor de log de verosimilitud y;

- el factor de inflación de la varianza (VIF), como prueba de multicolinealidad del modelo.

Se optó por incluir en el modelo seleccionado únicamente aquellas variables significativas ${ }^{8}$, lo que permitió mejorar los niveles de ajuste, mejorar la propiedad de balance de la regresión y no

\footnotetext{
${ }^{8}$ Variables cuyo valor p es inferior a 0,05 (significancia del 5\%), o inferiores a 0,1 (significancia del $10 \%$ ). 14 
incurrir en una sobreestimación del modelo, aspecto que no es aconsejable en la metodología PSM.

Como resultado de lo anterior, la ecuación probit que se utiliza para la estimación de los $P S$ presenta la siguiente estructura:

credito $=\beta 0+\beta 1 \times$ Zona $1+\beta 2 \times$ sectorprimario $+\beta 3$ sectorsecundario $+\beta 4$ inscrito $+\beta 5 \times$ EmpleoTotal $+\beta$ $6 x$ cobro_trans $+\beta 7$ xtelefono $+\beta 8$ xedu_secundariainc $+\beta 9$ xtiempo_1omas $+\beta 10$ xhoras 40 omas $+\varepsilon$ Donde:

- Bi es el coeficiente de cada una de las variables explicativas

- $\varepsilon$ corresponde al término de error

Esta regresión se caracteriza por haber sido la de mayor calidad estadística dentro del conjunto de expresiones estimadas. Por medio de los diferentes criterios mencionados más arriba existe certeza de la alta capacidad de la ecuación para determinar los puntajes de propensión.

En la Tabla $\underline{4}$ se pueden apreciar los resultados de la estimación de la ecuación probit:

Tabla 4

Estimación del modelo probit para la obtención de crédito por parte de los emprendedores de microempresas de los hogares en Costa Rica. Año 2015

\begin{tabular}{lccc}
\hline \multicolumn{1}{c}{ Variable } & Coeficiente & Error estándar & P>|z| \\
\hline Zona & $-2,2199$ & 0,0957 & 0,022 \\
Sector primario & 0,6191 & 0,1214 & 0,000 \\
Sector secundario & $-0,2022$ & 0,1213 & 0,095 \\
Inscrito & 0,6116 & 0,0895 & 0,000 \\
Empleo total & 0,1299 & 0,0316 & 0,000 \\
Cobro_trans & 0,3167 & 0,1078 & 0,003 \\
Teléfono & 0,3934 & 0,1502 & 0,009 \\
Edu_secundariainc & 0,2973 & 0,0928 & 0,001 \\
Tiempo_1 o más & 0,3265 & 0,1749 & 0,062 \\
Horas 40 o más & 0,3637 & 0,0856 & 0,000 \\
Constante & $-2,3477$ & 0,2304 & 0,000 \\
\hline N: & 1466,0000 & AIC: & 1198,392 \\
PROB>LR: & 0,0000 & BIC: & 1256,585 \\
PSEUDO R2: & 0,1886 & VIF Mean: & 2,750 \\
\hline
\end{tabular}

Fuente: elaboración propia con datos de INEC (2015c). Nota ${ }^{1}$ : El mayor valor de log de verosimilitud se obtuvo después de cuatro iteraciones, número bajo que refleja la buena calidad del modelo. Nota ${ }^{2}$ : Con ninguna de las variables se presentó problemas de multicolinealidad. 
Como se puede apreciar en la tabla, el modelo probit seleccionado alcanza una significancia global al obtener un $p$ valor conjunto igual a 0 . Con respecto a la significancia individual de las variables, en su mayoría son significativas al $1 \%, 5 \%$ y $10 \%$, a excepción de la variable zona que es significativa al $5 \%$ y $10 \%$, y de sector secundario y tiempo_1 o más, las cuales son significativas únicamente al $10 \%$.

En cuanto a los signos de los coeficientes, se obtuvieron los resultados esperados. No obstante, se opta por no brindar su interpretación, ya que lo verdaderamente importante en este estudio es que el modelo tenga un buen ajuste, aspecto que determina la calidad de los emparejamientos. Con la regresión previamente señalada se procede a la estimación del PS para cada una de las microempresas de la muestra. En la Tabla $\underline{5}$ se pueden verificar las principales estadísticas descriptivas obtenidas para los PS a utilizar:

Tabla 5

Estadísticas descriptivas de los PS estimados en la región de soporte común (grupo control y grupo de tratamiento)

\begin{tabular}{lrrr}
\hline & \multicolumn{1}{l}{ Total } & \multicolumn{1}{c}{ Control } & \multicolumn{1}{l}{ Tratados } \\
\hline N & 1361 & 1074 & 287 \\
PS Medio & 0,2090052 & 0,1714867 & 0,3494053 \\
Desviación estándar & 0,1755269 & 0,1452179 & 0,2057249
\end{tabular}

Región de soporte común $\quad[0,02929381 ; 0,99992431]$

Fuente: elaboración propia con datos de INEC (2015c).

Al crearse la región de soporte común, se impone la condición restrictiva que supone eliminar las observaciones que no se encuentren dentro de dicha área de traslape. Como resultado de ello se descartaron de la muestra propuesta 105 unidades de estudio ${ }^{9}$, las cuales pertenecían, en su totalidad, al grupo que no recibió crédito.

\section{Generación de emparejamientos y obtención de resultados}

Al estimarse los PS para cada una de las observaciones se realiza el emparejamiento entre las unidades tratadas y las de control, aspecto que permite seguidamente evaluar el impacto. Sin embargo, previo a ello es necesario recalcar el cumplimiento de las propiedades necesarias para el alcance de resultados robustos.

\footnotetext{
${ }^{9}$ La delimitación de la región de soporte común fue realizada por medio del programa econométrico STATA. 16 
Se destaca la creación de la región de soporte común, la cual ha sido mencionada en el apartado anterior y en concreto se pudo contemplar en la Tabla $\underline{5}$. En dicha zona serán emparejadas las observaciones tratadas con las de control y, posteriormente realizada la estimación del impacto.

En cuanto a la propiedad de balance, las estimaciones realizadas cumplen satisfactoriamente con dicha condición al nivel de significancia más veraz utilizado por el programa estadístico STATA $(0,01)$. Esta señala que las observaciones con el mismo valor de $P S$ tendrán similar distribución de características, permitiendo con ello simular una aleatorización en la asignación del tratamiento.

Para el desarrollo del emparejamiento entre las observaciones y la correspondiente estimación del $A T T$, según se indicó en la metodología, se desarrollaron los algoritmos de emparejamiento al vecino más cercano, estratificación y kernel. En la Tabla $\underline{6}$ se pueden apreciar los resultados alcanzados con los mismos.

Tabla 6

Efecto promedio de tratamiento para los individuos tratados según método de emparejamiento

\begin{tabular}{lccc}
\hline \multicolumn{1}{c}{ Modelo } & ATT & Error estándar & t \\
\hline Vecino más cercano & 0,291 & 0,171 & 1,699 \\
Estratificación & 0,210 & 0,149 & 1,403 \\
Kernel & 0,289 & 0,125 & 2,309 \\
\hline
\end{tabular}

Fuente: elaboración propia con datos de INEC (2015c).

Con el algoritmo de vecino más cercano el impacto del crédito en las ganancias es del 29,1\%, con una significancia del 5\%. En el caso del modelo kernel, el $A T T$ estimado es de $28,9 \%$, con una significancia en el resultado del $5 \%$. Con respecto a la técnica de emparejamiento por estratificación, el impacto resulta más bajo que el alcanzado con los algoritmos señalados previamente $(21 \%)$ y su nivel de significancia es del $10 \%$.

Es importante señalar que las diferencias entre los resultados de los modelos estimados pueden deberse a las particularidades de cada uno de los algoritmos y su forma de ajustarse a los datos disponibles, por lo que cada una de las estimaciones de ATT se toma como válida.

Ante lo anterior, el impacto medio del tratamiento en cuestión es $26,3 \%$, al ser este el promedio de los tres algoritmos realizados. Esto evidencia que los créditos obtenidos por los sujetos dueños de las microempresas tienen un impacto positivo y estadísticamente significativo en las ganancias de dichas unidades productivas. 


\section{Conclusiones y recomendaciones}

A partir de la evaluación del impacto realizada se demuestra que los créditos tienen un impacto positivo y estadísticamente significativo en las ganancias de las microempresas de hogares en Costa Rica. Este resultado es la primera evidencia en el país del efecto que tienen en las microempresas los préstamos obtenidos.

La obtención de crédito en las microempresas contribuye significativamente en su crecimiento de las ganancias. Para la población analizada, dicho crecimiento fue de $26 \%$, en promedio. Esto puede traducirse en mayor bienestar para los hogares productores vinculados, con lo cual se cumple el objetivo final de las microfinanzas.

Para los sujetos dueños de las microempresas es de alta relevancia la obtención de ayuda financiera, en 2015 el 48\% de dichas unidades productivas experimentó esta necesidad. Ante este hecho y el hallazgo alcanzado en el estudio, se insta a realizar una correcta gestión de créditos a este tipo de población, otorgando, de manera simultánea, capacitaciones que permitan a los grupos emprendedores adquirir los conocimientos básicos para el buen uso de los recursos brindados.

Otras conclusiones que se obtienen a partir de la investigación y destacan, por su alta relevancia ante la coyuntura nacional, son las siguientes:

- El nivel formativo de los sujetos emprendedores es menor que el de la población en general, predominando los bajos niveles de escolaridad. En concreto, el 66,7\% de personas emprendedoras presenta niveles de educación inferiores a "Secundaria completa", aspecto que les impide una movilización social más acelerada.

Contar con educación se traduce en más y mejores habilidades y capacidades, mayores niveles de productividad $\mathrm{y}$, con ello, un mayor capital humano que permitirá crecimiento en la producción y, por consiguiente, en los ingresos.

- Entre los sujetos emprendedores, más allá de iniciar actividades productivas con motivo de una oportunidad o tradición familiar, la principal razón para emprender fue la necesidad, la cual contempla no disponer de trabajo y el deseo de complementar el ingreso familiar. Esto refleja, en cierta medida, la situación económica en desventaja de un alto porcentaje de quienes conforman las microempresas de hogares.

Además, este punto se encuentra altamente relacionado con el que dicha población disponga, en su mayoría, de una baja escolaridad, aspecto que dificulta la inserción al mercado laboral.

18

Carmen Sánchez Retana

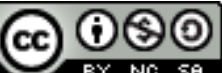

Revista Economía y Sociedad by Universidad Nacional is licensed under a CreativeCommons Reconocimiento-NoComercial- 
- Respecto a la vía por la cual se solicita apoyo, las instituciones estatales son altamente utilizadas. De las microempresas que solicitaron algún tipo de ayuda, el $40 \%$ lo hizo en un banco público. Además, de quienes solicitaron crédito el $51 \%$ lo hizo en la banca pública y un $15 \%$ en mutuales o cooperativas.

Lo anterior demuestra el importante vínculo entre la población y el Estado. Este hecho puede ser utilizado por el gobierno para implementar con mayor facilidad las políticas públicas dirigidas a esta población y, con ello, fortalecer el sistema empresarial, mejorar el empleo, aumentar los ingresos, reducir la pobreza y aumentar el crecimiento del país.

- Es importante el porcentaje de personas emprendedoras que a pesar de necesitar crédito no lo solicitan. Si bien el 48,4\% experimentó dicha necesidad, únicamente el 22,5\% lo solicitó. Además, de la totalidad de emprendedores que no solicitó crédito, el 63,1\% sí lo requería; pero, no lo solicitó por los siguientes motivos: desconocimiento de los procedimientos, cuantía o plazo no conveniente, los trámites asociados, creer que no se lo van a otorgar, el no sentirse bien adquiriendo una deuda o creer que no podría hacer frente al término amortizativo de esta misma.

Es alta la relevancia de otorgar educación financiera a nivel general, pero, sobre todo, en el sector informal de la economía. Aspecto que se acentúa al saber que "acceso a capacitaciones" es la segunda necesidad señalada por los grupos emprendedores.

Brindar facilidades educativas orientadas a las finanzas mejoraría la bancarización en la sociedad, permitiendo una mejor comprensión de los pros y contras de los créditos, los aspectos técnicos o de pago y los procedimientos de solicitud asociados, entre otros. Además, cultivarían en los emprendedores conocimientos para una adecuada gestión de los recursos crediticios a lo interno de la microempresa.

- Las evaluaciones de impacto enfocadas en microcréditos han sido escasas a nivel mundial y nulas en Costa Rica. Uno de sus principales motivos es la dificultad que experimentan las investigaciones en la creación del grupo de tratamiento y, sobre todo, del grupo de control (no existen los datos necesarios). En el trabajo realizado se hizo un esfuerzo por construir, con los datos disponibles, la muestra idónea que permitiera comparar ambos grupos y con ello obtener resultados robustos.

Sin embargo, es indispensable continuar avanzando en Costa Rica con la construcción de mayores y mejores bases de datos que permitan el desarrollo de estudios de relevancia en la toma de decisiones nacionales e institucionales. 
Se recomienda que en la ENHOPRO (hoy llamada Encuesta Nacional de Microempresas de los Hogares ENAMEH) se incluya mayor detalle de los créditos obtenidos por parte de los grupos emprendedores y de la actividad financiera en general. Dentro de dicho detalle se sugiere incluir variables que señalen las instituciones que conceden los créditos, así como la procedencia de los fondos utilizados para la concesión de estos mismos: SDB u otra fuente. La inclusión de esta última variable permitiría obtener resultados de gran relevancia para la toma de decisiones por parte del gobierno de Costa Rica, del SBD y de aquellas entidades vinculadas a éste.

Se recomienda aumentar los esfuerzos nacionales orientados en la solidificación del contexto microfinanciero, la inclusión financiera, el desarrollo de mayores regulaciones y la promoción de educación financiera entre la sociedad.

\section{Referencias}

Aroca, P., \& Hewings, G. (2009). Microcredit Impact Assesment: The Brazilian and Chilean Cases. Panorama Socioeconómico 27(39), 100-112. Recuperado de https://www.redalyc.org/pdf/399/39915006002.pdf

Augsburg, B., De Haas, R., Harmgart, H., \& Meghir, C. (2015). The Impacts of Microcredit: Evidence from Bosnia and Herzegovina. American Economic Journal: Applied Economics, 7(1), 183- 203. Recuperado de http://dx.doi.org/10.1257/app.20130272

Becker, S., \& Ichino, A. (2002). Estimation of average treatment effects bases on propensity scores. Stata Journal, 4(2), 358-377. Recuperado de https://ageconsearch.umn.edu/bitstream/116022/2/sjart st0026.pdf

García, J. (2004). Los micro préstamos como instrumentos de desarrollo en el sur: Especial referencia al ámbito latinoamericano. Madrid: CIDEAL.

Goldberg, N. (2005). Measuring the Impact of Microfinance: Taking Stock of What We Know. Washington, D.C.: Grameen Foundation USA. Recuperado de https://www.grameenfoundation.org/sites/default/files/resources/MeasuringImpact-of-Microfinance Nathanael Goldberg.pdf

Gujarati, D., \& Porter, D. (2010). Econometría. México: Mc Graw Hill Educación.

Heckman, J., Ichimura, H., \& Todd, P. (1998). Matching as an Econometric Estimator. Review of Economic Studies, 65, 261-294. Recuperado de http://www.uh.edu/ adkugler/Heckmanetal.pdf

20

Carmen Sánchez Retana

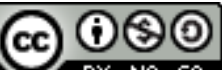

Revista Economía y Sociedad by Universidad Nacional is licensed under a CreativeCommons Reconocimiento-NoComercial- 
Henríquez, F. (2009). Microcrédito y su Impacto: Un acercamiento con datos chilenos. Working Paper, Banco Interamericano de Desarrollo, Washington, D.C. Recuperado de https://publications.iadb.org/es/publicacion/14627/microcredito-y-su-impacto-unacercamiento-con-datos-chilenos

Instituto Nacional de Estadística y Censos de Costa Rica (INEC). (2015a). encuesta nacional de hogares productores 2015. Resultados generales (ISSN 2215-4361). Recuperado de http://www.inec.go.cr/publicaciones?fuente tid=335

Instituto Nacional de Estadística y Censos de Costa Rica (INEC). (2015b). Encuesta nacional de hogares productores 2015. Resultados [Estadísticas]. Recuperado de http://www.inec.go.cr/encuestas/encuesta-nacional-de-hogares-productores

Instituto Nacional de Estadística y Censos de Costa Rica (INEC). (2015c). Encuesta nacional de hogares productores $2015 . \quad$ [Microdatos]. Recuperado de http://sistemas.inec.cr/pad4/index.php/catalog/157

Khandker, S., Koolwal, G., \& Samad, H. (2010). Handbook on Impact Evaluation. Quantitative Methods and Practices. Washington D.C., Estados Unidos: World Bank. Recuperado de

https://openknowledge.worldbank.org/bitstream/handle/10986/2693/520990PUB0 EPI11010fficial0Use00nly1.pdf

Larru, J. M. (2008). Las evaluaciones de impacto con asignación aleatoria y los microcréditos. Revista de Economía Mundial, 19, 33-62. Recuperado de https://www.redalyc.org/pdf/866/86601903.pdf

Monge, R., \& Rodríguez, J. (2012). El impacto de los servicios financieros y de capacitación en las MiPyME de Costa Rica. Costa Rica: Banco Interamericano de Desarrollo. Recuperado de

https://mba.americaeconomia.com/sites/mba.americaeconomia.com/files/el impa cto de los servicios financieros $y$ de capacitacion en las mipyme de costa ric a.pdf

Ordaz, J. (2009). México: Impacto de la educación en la pobreza rural. Centro de Estudios para América Latina (CEPAL), 105, 1-40. Recuperado de https://repositorio.cepal.org/bitstream/handle/11362/4883/S2009326 es.pdf?sequ ence $=1$ 
Rico, S. (2009). Microcrédito social: Una evaluación de impacto. Barcelona, España: Fundacion Caixa Catalunya. Recuperado de https://docplayer.es/13223764-Microcredito-socialuna-evaluacion-de-impacto.html

Rodríguez, M. (2012). Técnicas de evaluación de impacto: Propensity Score Matching y aplicaciones prácticas con Stata. España: Instituto de Estudios Fiscales. Recuperado de https://docplayer.es/40985975-I-f-estudios-fiscales-instituto-de-tecnicas-deevaluacion-de-impacto-propensity-score-matching-y-aplicaciones-practicas-constata.html

Rosembaum, P., \& Rubin, D. (1983). The central role of the propensity score in observational studies for causal effects. Biometrika, 70(1), 41-55. Recuperado de http://www.stat.cmu.edu/ ryantibs/journalclub/rosenbaum 1983.pdf

Rosembaum, P., \& Rubin, D. (1984). Reducing Bias in Observational Studies Using Subclassification on the Propensity Score. Journal of the American Statistical Association, 79, 516-524. Recuperado https://www.jstor.org/stable/2288398?seq=1\#page scan tab contents

Sanhueza, M. (2011). Microempresa y microfinanzas como instrumento de desarrollo local. Evaluación de impacto del microcrédito en la región de La Araucanía, Chile (Tesis doctoral). Recuperado de https://repositorio.uam.es/handle/10486/5981

Sánchez, C. (2015a). Impacto de la educación en la pobreza de la zona rural en Costa Rica. San José, Costa Rica: Universidad de Costa Rica.

Sánchez, C. (2015b). Evaluación de impacto de la educación sobre la pobreza en Costa Rica (un análisis para educación secundaria y post-secundaria). Revista de Ciencias Económicas, 33(2), 10-23. doi: http://dx.doi.org/10.15517/rce.v33i2.22608 
Anexo 1

Descripción de las variables consideradas en las estimaciones

\begin{tabular}{|c|c|c|}
\hline Variable & Definición & Detalle \\
\hline \multicolumn{3}{|l|}{ Variable de impacto } \\
\hline Inganancias & $\begin{array}{l}\text { Representa el crecimiento de las } \\
\text { ganancias }\end{array}$ & $\begin{array}{l}\text { Logaritmo natural de las ganancias de la } \\
\text { microempresa }\end{array}$ \\
\hline \multicolumn{3}{|c|}{ Variable de tratamiento } \\
\hline crédito & Obtención de crédito & $\begin{array}{l}0: \text { no recibió crédito } \\
\text { 1: recibió crédito }\end{array}$ \\
\hline \multicolumn{3}{|c|}{ Características de la actividad } \\
\hline Zona & Zona (urbana o rural) & $\begin{array}{l}\text { 0: rural } \\
\text { 1: urbano }\end{array}$ \\
\hline Sector primario & $\begin{array}{l}\text { Desarrollo de actividades en el sector } \\
\text { primario }\end{array}$ & $\begin{array}{l}\text { 0: las actividades que se realizan no } \\
\text { corresponden al sector primario } \\
\text { 1: se realizan actividades del sector primario }\end{array}$ \\
\hline Sector secundario & $\begin{array}{l}\text { Desarrollo de actividades en el sector } \\
\text { secundario }\end{array}$ & $\begin{array}{l}\text { 0: las actividades que se realizan no } \\
\text { corresponden al sector secundario } \\
\text { 1: se realizan actividades del sector } \\
\text { secundario }\end{array}$ \\
\hline Sector & $\begin{array}{l}\text { Sector productivo al que pertenece la } \\
\text { microempresa }\end{array}$ & $\begin{array}{l}1 \text { Agropecuario } \\
2 \text { Industria } \\
3 \text { Comercio } \\
4 \text { Servicios }\end{array}$ \\
\hline \multicolumn{3}{|c|}{ Características del negocio } \\
\hline TipoMicro & $\begin{array}{l}\text { Tipo de microempresa, según sea } \\
\text { empleador o no }\end{array}$ & $\begin{array}{l}\text { 0: Cuenta propia } \\
\text { 1: Empleador }\end{array}$ \\
\hline inscrito & Inscripción legal del negocio & $\begin{array}{l}\text { 0: el negocio no está inscrito legalmente } \\
\text { 1: el negocio está inscrito legalmente }\end{array}$ \\
\hline SalAsig & $\begin{array}{l}\text { El emprendedor tiene asignado un } \\
\text { salario por su trabajo en el negocio }\end{array}$ & $\begin{array}{l}0: \text { no tiene asignado } \\
\text { 1: sí tiene asignado }\end{array}$ \\
\hline Socios & Tenencia de socios & $\begin{array}{l}\text { 0: Emprendedor no tiene socios } \\
\text { 1: Emprendedor tiene socios }\end{array}$ \\
\hline NumSocios & $\begin{array}{l}\text { Número de socios que tiene el } \\
\text { emprendedor }\end{array}$ & Variable continua \\
\hline EmpleoTotal & $\begin{array}{l}\text { Tamaño del empleo generado } \\
\text { incluyendo al dueño }\end{array}$ & Variable continua \\
\hline ubi_establecimiento & Principal ubicación de la actividad & $\begin{array}{l}\text { 1: espacio o local } \\
\text { 2: dentro de su casa } \\
\text { 3: finca agropecuaria } \\
\text { 4: a domicilio } \\
\text { 5: en vía pública } \\
\text { 6: otros }\end{array}$ \\
\hline Compradores & Tipo de clientes & $\begin{array}{l}\text { 0: público en general } \\
\text { 1: Estado, comercio, empresas, cooperativas, } \\
\text { otros. }\end{array}$ \\
\hline
\end{tabular}




\begin{tabular}{|c|c|c|}
\hline Variable & Definición & Detalle \\
\hline Venta con pedido & $\begin{array}{l}\text { Generación de ventas únicamente con } \\
\text { pedido previo }\end{array}$ & $\begin{array}{l}\text { 1: Venta únicamente con pedido o encargo } \\
0: \text { otro }\end{array}$ \\
\hline MotivEmprend & Motivo de emprendimiento & $\begin{array}{l}1 \text { Necesidad } \\
2 \text { Oportunidad } \\
3 \text { Tradición }\end{array}$ \\
\hline FormaCobro & Principal forma de cobro & $\begin{array}{l}0: \text { A crédito } \\
\text { 1: De contado }\end{array}$ \\
\hline Factura & Entrega de factura timbrada a clientes & $\begin{array}{l}\text { 0: no entrega factura timbrada } \\
\text { 1: sí entrega factura timbrada }\end{array}$ \\
\hline Cobro_trans & $\begin{array}{l}\text { Pago de clientes por medio de } \\
\text { transferencia electrónica }\end{array}$ & $\begin{array}{l}\text { 0: El pago de los clientes no se realiza por } \\
\text { medio de transferencia electrónica } \\
\text { 1: El pago de los clientes sí se realiza por } \\
\text { medio de transferencia electrónica }\end{array}$ \\
\hline Ctabancaria & $\begin{array}{l}\text { Disposición de cuenta bancaria para } \\
\text { uso exclusivo del negocio }\end{array}$ & $\begin{array}{l}\text { 0: no dispone de cuenta bancaria para uso } \\
\text { exclusivo del negocio } \\
\text { 1: sí dispone de cuenta bancaria para uso } \\
\text { exclusivo del negocio }\end{array}$ \\
\hline Internet & Acceso a internet & $\begin{array}{l}0: \text { no tiene acceso a internet } \\
\text { 1: tiene acceso a internet }\end{array}$ \\
\hline Teléfono & Uso de teléfono fijo o móvil & $\begin{array}{l}\text { 0: no se utiliza el teléfono fijo o móvil } \\
\text { 1: sí se utiliza el teléfono fijo o móvil }\end{array}$ \\
\hline \multicolumn{3}{|c|}{ Características del sujeto emprendedor } \\
\hline Edad & Edad & Variable continua \\
\hline Sexo & Sexo & $\begin{array}{l}\text { 0: mujer } \\
\text { 1: hombre }\end{array}$ \\
\hline JefeHogar & $\begin{array}{l}\text { Relación de jefatura de la persona } \\
\text { dueña del negocio }\end{array}$ & $\begin{array}{l}0: \text { No es jefe de hogar } \\
\text { 1: Es jefe de hogar }\end{array}$ \\
\hline fuente_ingreso & $\begin{array}{l}\text { Actividad como principal fuente de } \\
\text { ingreso del hogar }\end{array}$ & $\begin{array}{l}\text { 0: La actividad en estudio no es la principal } \\
\text { fuente de ingreso del hogar } \\
\text { 1: La actividad en estudio es la principal } \\
\text { fuente de ingreso del hogar }\end{array}$ \\
\hline edu_secundariainc & $\begin{array}{l}\text { Nivel educativo del dueño de la } \\
\text { microempresa }\end{array}$ & $\begin{array}{l}\text { 0: Educación primaria o menos } \\
\text { 1: Secundaria incompleta o más }\end{array}$ \\
\hline tiempo 1 o más & $\begin{array}{l}\text { Tiempo que se ha desarrollado la } \\
\text { actividad }\end{array}$ & $\begin{array}{l}\text { 0: menos de un año } \\
\text { 1: Un año o más }\end{array}$ \\
\hline Horas 40 o más & $\begin{array}{l}\text { Dedicación de horas por semana al } \\
\text { negocio }\end{array}$ & $\begin{array}{l}\text { 0: dueño dedica al negocio menos de } 40 \\
\text { horas por semana } \\
\text { 1: dueño dedica al negocio } 40 \text { horas o más } \\
\text { por semana }\end{array}$ \\
\hline Anos & $\begin{array}{l}\text { Tiempo de desarrollo de la actividad en } \\
\text { años }\end{array}$ & Variable continua \\
\hline
\end{tabular}

Fuente: elaboración propia.

24 